\title{
¿Qué retos impone la economía digital a la próxima administración?
}

\section{Octavio Islas Carmona Fernando Gutiérrez Cortés ${ }^{1}$}

ARA PODER RESPONDER a LA PREGUNTA que hemos formulado, en primera instancia procederemos a definir qué significa el término "Comercio Electrónico". Para tal efecto hemos recurrido a las autorizadas opiniones de reconocidos consultores de los Estados Unidos, quienes han asesorado en materia de Comercio Electrónico a un amplio número de instituciones. Enseguida referiremos cuáles son los modelos de Comercio Electrónico que actualmente se están ensayando en el mundo, los cuales comprenden: operaciones comerciales en línea de empresas con empresas (B2B); transacciones en línea de empresas con consumidores (B2C); operaciones comerciales y de servicios del consumidor al negocio (C2B); intercambio de bienes y servicios de consumidor a consumidor ( $\mathrm{C} 2 \mathrm{C}$ ); operaciones comerciales y de servicios de empresas con el gobierno (B2G), servicios del gobierno a consumidores y ciudadanos (G2C); transacciones en línea de gobierno a gobierno (G2G).

A partir de 1997, el desarrollo de operaciones de Comercio Electrónico por Internet ha registrado un sostenido crecimiento. Actualmente más de 332 millones de perso-

1. Investigadores del Proyecto Internet del Tecnológico de Monterrey, Campus Estado de México. Fernando Gutiérrez Cortés (27 años) realizó la Licenciatura en Ciencias de la Comunicación en el Tecnológico de Monterrey, Campus Estado de México y la Maestria en Administración de Tecnologías de Información (MATI), en la Universidad Virtual del Tecnológico de Monterrey. Actualmente cursa una segunda maestria, también en el Tecnológico de Monterrey, en Comercio Electrónico. Además realizó un Diplomado en Comunicación Política en el Instituto Nacional de Administración Pública (INAP). Octavio Islas Carmona (40 años). Sociólogo, realizó la Maestría en Comunicación en la Universidad Iberoamericana y el Doctorado en Ciencias Sociales en la Universidad La Salle. Actualmente realiza una segunda maestría, en Administración en Tecnologías de la Información, en el Tecnológico de Monterrey. También realizó un Diplomado en Comunicación Política en el Instituto Nacional de Administración Pública (INAP). En el Proyecto Internet del Tecnológico de Monterrey, Campus Estado de México, Octavio Islas y Fernando Gutiérrez han coordinado el desarrollo de 
nas ya tienen acceso a la gran red de redes. El impacto de Internet en las sociedades contemporáneas de ninguna manera se limita al ámbito estrictamente informativo. Internet ha impuesto aceleradas y profundas transformaciones en los mercados internacionales, impulsando el desarrollo de nuevos modelos de negocios, los cuales forman parte de la llamada "economía digital".

Del nuevo escenario cultural que han impuesto sobre las sociedades contemporáneas las avanzadas tecnologías de información asociadas a Internet, se desprende el desarrollo de un nuevo tipo de tejido social, una nueva división del trabajo, nuevos parámetros en materia de productividad, nuevos principios de actuación para el Estado, nuevos criterios de estratificación social sustentados en el acceso y en el uso de las tecnologías, nuevos escenarios en la geopolítica de la información, así como nuevas formas y relaciones de conocimiento. En el año 2003 seguramente se habrá consumado la fusión de la televisión e Internet, derivándose de tal hibridación el advenimiento de un nuevo y complejo medio de comunicación, el cual definitivamente habrá de contribuir a extender el desarrollo del Comercio Electrónico en el mundo.

En los próximos años México podría convertirse en el principal eje de desarrollo del Comercio Electrónico en América Latina. Por tal motivo nos parece indispensable incorporar al próximo Plan Nacional de Desarrollo un apartado dedicado al tema del Comercio Electrónico, reparando en el sentido estratégico que podría admitir un programa gubernamental orientado a impulsar la capacidad exportadora de nuestras micro, pequeñas y medianas empresas, brindándoles toda la asesoría necesaria.

A marchas forzadas la LVII Legislatura ya introdujo las primeras iniciativas orientadas a regular el desarrollo del Comercio Electrónico en México. Tales iniciativas todavía son insuficientes. La próxima administración tendrá que diseñar estrategias definidas en materia de Comercio Electrónico, pues éste podría generar un considerable número de divisas para el pais, derivándose la creación de nuevas fuentes de empleo para miles de mexicanos. Por lo anterior resulta indispensable extender el marco normativo que habrá de regular el desarrollo del Comercio Electrónico en México.

algunos de los principales sitios www en México, como es el caso de la Presidencia de los Estados Unidos Mexicanos (1996); PEMEX Corporativo (1996 y 1998); Cámara de Senadores (1998-1999), y desde 1997 a la fecha, la Cámara de Diputados. También crearon la primera revista electrónica de América Latina dedicada a abordar temas de comunicación: Razón y Palabra. Proyecto Internet se encarga del desarrollo y la administración del www del Consejo Nacional para la Enseñanza y la Investigación de las Ciencias de la Comunicación (CONEICC), así como las páginas www de la Revista Mexicana de Comunicación (http://www.fundacionbuendia.org.mx) y las páginas www de la Red de Investigación de la Comunicación Compleja (http://www.mexicocomunica.org.mx) Sus correos electrónicos son: fgutierr@campus.cem.itesm.mx y oislas@campus.cem.itesm.mx El URL de Proyecto Internet es http://www.comunicación.org.mx. 


\section{¿Qué es el Comercio Electrónico?}

Moira Jacobs, quien funge como responsable de una oficina del Departamento de Comercio del gobierno de los Estados Unidos, la cual se encarga de proporcionar servicios integrales de consultoría en materia de Internet y Comercio Electrónico a través de más de 100 centros, a micro, pequeñas y medianas empresas estadounidenses interesadas en exportar en línea sus mercancías y servicios, define el Comercio Electrónico como: "cualquier transacción entre compradores y vendedores a través de medios electrónicos".

Gregory Martín, presidente de Corrillian, firma dedicada a proporcionar servicios de consultoría estratégica en materia de Internet, considera que el Comercio Electrónico es: "un emergente ambiente de negocios derivado de la convergencia de las computadoras y las comunicaciones". 3

Vadim Levitin, consultor de la compañía ecRx.com, refiere la siguiente definición de Comercio Electrónico, la cual procede de los reconocidos consultores de The Gartner Group: "el Comercio Electrónico es un envolvente conjunto de herramientas de tecnologías de información, así como estrategias de negocios destinadas a favorecer la realización de prácticas comerciales de forma electrónica". ${ }^{4}$

Hoy el término "Comercio Electrónico" suele utilizarse para designar las transacciones comerciales en línea que personas, empresas, organizaciones, e inclusive gobiernos realizan entre sí. El Comercio Electrónico de ninguna manera se restringe al desarrollo de operaciones comerciales en línea que realizan empresas con empresas (B2B) o empresas con consumidores (B2C). El Comercio Electrónico también comprende el desarrollo de operaciones comerciales y de servicios en Internet del consumidor al negocio (C2B); intercambio de bienes y servicios de consumidor a consumidor (C2C); operaciones comerciales y de servicios de empresas con el gobiemo (B2G); servicios del gobierno a consumidores y ciudadanos (G2C); transac-

2. Respecto a los resultados del trabajo que han venido realizando los referidos centros, Moira Jacobs destacó que un total de 427,000 pequeños negocios iniciaron operaciones comerciales en línea durante el periodo comprendido entre abril de 1998 y abril de 1999. Véase Moira Jacobs: "International E-Business Oportunities and Challenges: A Small Business Perspective". Ponencia presentada en The 1999 e-Business Opportunities, Challenges, and Winning Strategies: Universidad de Montana en Missoula y The Montana World Trade Center, Estados Unidos, 2-5 de agosto de 1999.

3. Greg Martin: "Internet Banking in Exploding e-Space: Customer Compliant or Customer Complacent". Ponencia presentada en The 1999 e-Business Conference, Universidad de Montana en Missoula y The Montana World Trade Center, Estados Unidos, 2-5 de agosto de 1999.

4. Vadim Levtin: "Getting Started in Cyperspace: Reviewing the Basics". Ponencia presentada en The 1999 e-Business Conference. Universidad de Montana en Missoula y The Montana World Trade Center, Estados Unidos, 2-5 de agosto de 1999. 
ciones de gobierno a gobierno (G2G). A continuación procederemos a enunciar algunas de las principales características de los modelos de Comercio Electrónico que hemos referido.

\section{Modelo de Comercio Electrónico de operaciones de empresas a empresas (B2B)}

Este modelo comprende el desarrollo de operaciones comerciales y de servicios entre empresas, a través de sistemas de Internet o Extranets. De acuerdo con Mario de la Garza, especialista en el área de Cibermarketing, ${ }^{5}$ el modelo de negocios en línea B2B promueve el desarrollo de operaciones de intercambio comerciales en línea entre dos o más empresas, agilizándose el procesamiento de las peticiones de compra-venta, planificándose la producción conforme a los tiempos más adecuados para cada uno de los negocios involucrados en las operaciones y verificándose automáticamente los estados de los inventarios.

Además es posible trabajar de forma vertical, conectándose dos negocios en línea, o de forma horizontal, con todos los negocios que están involucrados en una operación comercial, permitiendo también el concentrar las transacciones, instalar sistemas "just in time", desarrollar sistemas de pagos electrónicos de nómina, conferir un decidido impulso a relaciones que impliquen transacciones entre organizaciones y agilizar la evolución natural de las aplicaciones de intercambio electrónico de datos. En México importantes empresas de la industria farmacéutica, el sector automotriz, electrodomésticos, industria química, industria del vestido y la industria de la computación ya realizan un considerable número de transacciones en línea a través de modelos de Comercio Electrónico B2B.

\section{Modelo de Comercio Electrónico \\ de empresas a consumidores (B2C)}

Este modelo implica el desarrollo de las estrategias y de la infraestructura necesaria para efectuar la promoción y la venta en línea de productos y servicios que ofrecen empresas a potenciales consumidores.

5. Mario de la Garza es autor del ensayo "Internet y Comercio Electrónico", el cual forma parte del libro: Internet: El medio de comunicación Inteligente. Octavio Islas y Fernando Gutiérrez (comps.), el cual próximamente publicará el Grupo Patria Cultural. 
La formidable expansión de este modelo de negocios en línea representa una definitiva contribución al desarrollo de los mercados abiertos y extendidos. En las empresas este modelo de negocios favorece la aceleración del ciclo de compra, impulsando el desarrollo de transacciones directas y personalizadas en línea con el cliente.

Evidentemente de este modelo se impone la necesidad de emprender profundas transformaciones en el tipo de servicios que deberá realizar la empresa después de haberse efectuado la venta para mantener la lealtad y la preferencia de los consumidores.

Prácticamente cualquier empresa que disponga de la infraestructura para ingresar en este ambiente de negocios podrá comercializar sus mercancías y servicios en Internet. Las operaciones comerciales en línea de Ticketmaster, Amazons e inclusive de portales como "Todito.com" corresponden a este modelo de negocios en línea.

\section{Modelo de Comercio Electrónico}

de consumidores a negocios (C2B)

El modelo C2B también representa una significativa contribución al desarrollo de mercados abiertos y extendidos, pues supone que cualquier trabajador independiente se encuentre en condiciones de poder ofrecer en línea sus servicios o bienes a empresas o a compradores potenciales.

Consumidor a consumidor (C2C)

De este modelo de negocios en línea procede el trueque e intercambio de productos y servicios entre consumidores. Este modelo estimula el desarrollo de transacciones directas entre personas físicas, quienes para tal efecto emplean las más avanzadas tecnologías a costos sumamente módicos.

Algunas empresas virtuales, reconociendo las oportunidades de negocios que se desprenden de este modelo, decididamente han incursionado en la red para promover sistemas de subastas o remates de mercancías en línea, por ejemplo: Mercado Libre y De remate.com. ${ }^{6}$

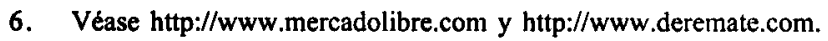


Negocio a gobierno (B2G)

Comprende la venta en línea de productos y servicios que distintas empresas ofrecen al gobierno. Consideramos que si nuestro gobierno rápidamente llega a comprender la importancia que podría adquirir en los próximos años este modelo de negocios en línea, seguramente estará en condiciones de poder obtener mejores, precios, identificar mejores proveedores, encontrar mejores productos y servicios y negociar mejores condiciones de pago.

\section{Gobierno a consumidor (G2C)}

Este modelo impone profundas transformaciones en las relaciones Estado-sociedad. El gobierno paulatinamente habrá de desempeñarse en Internet como un auténtico proveedor de relevante información destinada a la ciudadanía.

Para tal efecto, en primera instancia el Estado deberá acceder a la condición de un Estado integrado. Tal escenario supone la necesidad de integrar coherentemente todos los servicios de información en línea con que cuenta el Estado. En ese proceso, el gobierno decididamente habrá de involucrarse en el desarrollo de una profunda reforma administrativa, la cual desbordará el estricto ámbito de intervención del Estado en el ciberespacio para extenderse a otros escenarios. El Estado extendido tendrá que someterse a nuevos principios de actuación en un considerable número de asuntos y cuestiones públicas. De las nuevas relaciones de información en las cuales descansará la relación Estado-sociedad se derivarán plebiscitos cotidianos. El Estado tendrá pues que transformarse.?

La inteligente aplicación de este modelo le permitirá al Estado el simplificar un considerable número de trámites que diariamente tiene que realizar todo ciudadano, abriendo nuevos conductos para la recaudación fiscal, erradicándose algunas de las desafortunadas prácticas de corrupción que suelen realizar burócratas menores “ - como dificultar el acceso a la información o complicarle a la ciudadanía el desarrollo de un considerable número de trámites-. Un interesante ejemplo de este

7. La revista Gobierno Digital, la cual desafortunadamente ya no se publica, de alguna manera contribuyó a sensibilizar a directores de informática y a responsables de las áreas de comunicación y relaciones públicas de algunas dependencias públicas respecto a la importancia que admite el desarrollo de una positiva cibercultura en la administración pública. Sobre este tema particularmente recomendamos consultar el número 24 de Gobierno Digital, el cual llevó por título "Reinventar el gobierno en México"; y el número 26: "Informática para rediseñar al gobierno". 
modelo lo representa el Sistema de Declaración de Impuestos en Internet de la Secretaría de Hacienda.

Gobierno a gobierno (G2G)

En un futuro no muy distante, el desarrollo de operaciones comerciales en línea entre gobiernos será una práctica frecuente.

\section{El tránsito hacia la "economía digital"}

El desarrollo exponencial del Comercio Electrónico en el mundo, en primera instancia procede de la formidable expansión que alcanzó Internet a partir de la explotación comercial que admitió la World Wide Web (www) a mediados de la década de $1990 .^{8}$ Por tal motivo el año de 1995 ha sido considerado como el "año de Internet".

Las graves turbulencias políticas que a los mexicanos nos deparó el año de 1994, explican la acentuada indiferencia que mostraban en 1995 amplios sectores de nuestra sociedad respecto al tema de Internet. ${ }^{9}$ Particularmente en el sector público predominaba una especie de apatía hacia todo aquello que pudiese estar relacionado con el desarrollo de Internet en México. Inclusive es admisible aseverar que un amplio número de servidores públicos entonces suponía que Internet era un recurrente tema de la literatura de ciencia ficción.

Como ejemplo del pobre interés que en sus inicios mostraba la administración del presidente Ernesto Zedillo hacia los asuntos relativos al tema de Internet, ${ }^{10}$ basta referir que el llamado "Programa de Desarrollo Informático", elaborado por la Aca-

8. En 1990, el científico inglés Tim Berners-Lee desarrolló un nuevo sistema de hipertexto en el Laboratorio de Física de Partículas (CERN), de Suiza. Ese sistema se conocería como World Wide Web (www). La idea de crear la www se remite a una necesidad muy particular de Tim Berners Lee: integrar su agenda, su indice telefónico y diversos documentos personales. Un programa desarrollado por Tim Berners Lee en 1980 se convirtió en la base técnica del web, la cual al principio estaba restringida al uso interno de investigadores del CERN, pero que pronto fue incorporado por otros investigadores externos.

9. En 1995, millones de mexicanos todavía no conseguiamos reponernos de los graves sobresaltos que nos deparó el año de 1994, al cual Carlos Fuentes agudamente calificó de "año peligroso" Véase Carlos Fuentes: Nuevo Tiempo Mexicano, Editorial Aguilar, México. Particularmente el capítulo séptimo: "El 94. Diario de un año peligroso".

10. En diciembre de 1995 , NIC-México sólo consignaba 12 dominios “.gob.mx", de un total de 326 dominios ".mx". 
demia Nacional de la Informática y derivado del "Plan Nacional de Desarrollo", no solo no contempló ningún tipo de estrategia definida por parte del Estado en materia de Internet. Como si se tratase de una palabra maldita, el referido plan ni siquiera consignó en una sola ocasión la palabra Internet."

Para la administración del Presidente Ernesto Zedillo, Internet sencillamente no fue considerado como un asunto del cual debería ocuparse el Estado. La ausencia de elementales estrategias del Estado en materia de comunicación social en el ciberespacio las hemos señalado en repetidas ocasiones. Simplemente podemos concluir que ninguno de los funcionarios que Zedillo improvisó durante su administración como responsables de la Dirección General de Comunicación Social de la Presidencia de la República —operadores políticos ajenos al ejercicio profesional de la comunicación social- alcanzó a advertir la importancia de establecer estrategias definidas en materia de comunicación social del Estado en el ciberespacio.

Pese a las amargas críticas de sus detractores, hoy Internet es la forma más rápida y sencilla de facilitar y obtener información pues, como afirma Gerhard Schweigker:

Si inicialmente hubo algunas dudas en cuanto a la utilidad de la red (se hablaba de pocas informaciones útiles, mucha anarquía y poca sistematización), hoy ha desarrollado una dinámica propia que hace que los gobiernos, empresas, asociaciones, universidades, instituciones científicas y los propios medios clásicos ya no puedan estar ausentes en la red. Al mismo tiempo, buscadores cada vez más sofisticados y exactos facilitan los medios necesarios para que el usuario pueda orientarse en una avalancha de datos. ${ }^{12}$

Del formidable desarrollo que ha alcanzado Internet indudablemente se han derivado importantes transformaciones en el orden informativo internacional. Respecto a las repercusiones de Internet sobre el escenario internacional de la información, AlejandroVázquez-Vela Duhalt, Director General de Canales de Televisión del Grupo MVS Comunicaciones, afirma lo siguiente:

En esta era globalizadora en la cual los gigantes se unen, se funden o simplemente se alian en joint ventures para mejorar su posición en el mercado, ninguna megafusión ha tenido el impacto que aquella anunciada el martes 11 de enero entre American

11. Véase: http://ww.inegi.gob.mx/informatica/espanol/informatica.html.

12. Véase: Gerhard Schweigker: Política Internacional: ¿Un mundo feliz?, en Josef Thesing y Frank Priess (Editores): Globalización, Democracia y Medios de Comunicación. Konrad-Adenauer Stiftung, Argentina, 1999. p. 366. 
Online y Time Warner. Lo sorprendente de la transacción no proviene solamente de la magnitud de las cifras (165,000 millones de dólares), ni por el hecho de que fuera AOL, una empresa relativamente nueva, con tan sólo 12,100 empleados y un EBITDA (ganancias antes de intereses, impuestos, depreciación y amortización) de únicamente 1,520 millones de dólares, la que compró en realidad a Time Warner, un consorcio de medios tradicional, con más de 70,000 empleados y un EBITDA de 8,440 millones de dólares. Lo que en realidad sorprendió a los analistas es que esta megafusión, la más grande de la historia, es la primera que vino a cimbrar desde sus cimientos a las Mecas de varias industrias: Hollywood, centro mundial del entretenimiento; Silicon Valley y la construcción de la superautopista de la información; Wall Street y el mundo de las finanzas; y Madison Avenue, centro neurálgico de la publicidad mundial. ${ }^{13}$

Como acertadamente destaca Alejandro Vázquez-Vela, el impacto de Internet en las sociedades contemporáneas ya no se limita al ámbito estrictamente informativo. Internet también ha impuesto aceleradas y profundas transformaciones en el mercado mundial, derivándose el consecuente desarrollo de la llamada "economía digital".

Respecto al impacto de Internet sobre la apertura y la extensión de los mercados actuales, Stan Davis y Christopher Meyer - investigadores del Ernst \& Young Center for Business Innovation de los Estados Unidos-, afirman que tres factores han incidido directamente en el desarrollo de la "economía digital": la velocidad, la conectividad y la revalorización de lo intangible. ${ }^{14}$

En cuanto al factor de la velocidad, Bill Gates ${ }^{15}$ afirma que si la calidad fue considerada como el gran "paradigma" de la productividad organizacional durante la década de 1980, y los procesos de "reingería" adquirieron similar relevancia para las empresas durante la década de 1990, en las organizaciones del 2000 habremos de considerar a la velocidad como el nuevo fundamento de la competitividad: "If the 1980 s were about quality and the 1980 s were about reengineering, the 2000 s will be about velocity". ${ }^{16}$

13. Alejandro Vázquez-Vela Duhalt es autor del ensayo "Televisión e Internet", el cual forma parte del libro: Internet: El medio de comunicación Inteligente. Octavio Islas y Fernando Gutiérrez (comps.), que próximamente publicará Grupo Patria Cultural.

14. Véase: Davis Stan y Meyer Christopher Meyer (1999): BLUR The spped of change in the connected economy, Warner Books, USA.

15. La impresionante fortuna que ha logrado acumular el polémico empresario y genial programador Bill Gates, admite en sí misma el carácter de perfecta metáfora del sentido y significado de la emergente economía digital, y de la nueva racionalidad económica de nuestros nuevos "tiempos modernos". Tanto Gates como Steve Jobs - el fundador de Apple--, fueron distinguidos miembros de la llamada "Generación Atari", la cual dedicó una considerable cantidad de horas al uso de la Commodore 64.

16. Véase Bill Gates: Business @ the speed of thought. Using a digital nervous system. Warner Books, USA.1999. 
¿Qué retos impone la economía digital a la próxima administración?

En las llamadas organizaciones "inteligentes" del nuevo milenio - afirma Gates, las avanzadas tecnologías de información asociadas a Internet habrán de erigirse como sistemas nerviosos digitales de las mismas. La contribución de los sistemas nerviosos digitales a la dinámica general de la organización se establece en tres dimensiones: pensamiento, reacción y adaptación. Lógicamente los complejos sistemas nerviosos digitales también podrán destinarse a la realización de funciones panópticas, vigilando y sancionando la actuación de las organizaciones. ${ }^{17} \mathrm{La}$ inteligente aplicación de la información que procesan las organizaciones a través del inteligente empleo de las avanzadas tecnologías de información les permitirá acceder a la condición de organizaciones integradas, para posteriormente trascender a la condición de organizaciones extendidas.

Hoy el tema de la conectividad ha trascendido el estricto ámbito operativo y reflexivo de la informática, las redes y los sistemas para convertirse también en un complejo objeto de estudio para los científicos sociales. Del nuevo escenario cultural que han impuesto las avanzadas tecnologías de información sobre las sociedades contemporáneas se desprende el desarrollo de un nuevo tipo de tejido social, una nueva división del trabajo, nuevos parámetros de productividad, nuevos principios de actuación para el Estado, nuevos criterios de estratificación social, nuevos escenarios de la información, nuevas formas y relaciones de conocimiento.

Davis y Meyer afirman que como consecuencia del incremento de la velocidad en el mundo de los negocios, las organizaciones competitivas —obligadamente cableadas-, se verán en la necesidad de operar y cambiar frecuentemente su forma de operar en "tiempo real", si efectivamente éstas desean ajustarse a las nuevas exigencias competitivas que les impondrá su mismo entorno. La conectividad supone la integración de productos, personas, compañías y naciones. La conectividad se proyecta como premisa fundamental de la competitividad de las organizaciones integradas.

En cuanto al tema de los intangibles, Davis y Meyer afirman que cualquier oferta simultáneamente admite dos parámetros de valor: un valor económico tangible y uno intangible. El sentido del valor intangible crece de forma más acelerada en las sociedades abiertas a los mercados virtuales. Al nuevo mercado internacional, resultante de tales transformaciones, los autores denominan "the BLUR".

En las sociedades de la información el compartir conocimientos produce valor. Ante las nuevas exigencias, las instituciones convencionales, acostumbradas a concentrar conocimientos para establecer y afirmar su autoridad, estarán en graves predicamentos.

17. Véase M. Foucault: Vigilar y castigar. Siglo xxI, México, 1985. 


\section{Las estadísticas de la "economía digital"}

Conforme a estimaciones realizadas por el Departamento de Asuntos Exteriores y de Comercio del gobierno de Australia, en 1997 las operaciones comerciales que se realizaron a través de Internet representaron un total de 5 billones de dólares. En cambio la firma IDC, dedicada a realizar servicios de investigación en el sector de las telecomunicaciones, la informática, Internet y el Comercio Electrónico, estimó en 10 billones de dólares el monto de las ventas en línea que se generaron durante ese mismo año. ${ }^{18}$

Independientemente de las diferencias que pueden advertirse en las cifras que ofrecen las referidas instituciones, lo cierto es que la mayor parte de las autorizadas firmas de consultoría coincide en señalar que a partir de 1997 el Comercio Electrónico por Internet adquirió un notable crecimiento, pues en 1999, de acuerdo con información que Moira Jacobs atribuyó a la empresa ActivMedia, las ventas en línea ascendieron durante ese año a la cantidad de 180 billones de dólares. ${ }^{19}$

De acuerdo con estimaciones del Departamento de Asuntos Exteriores y de Comercio del gobierno de Australia, en el año 2001 se realizarán ventas en línea por 150 billones de dólares. En cambio la firma IDC ha estimado en 220 billones de dólares las ventas que se harán a través de Internet en el año $2001 .^{20}$

En el año 2003 seguramente se habrá consumado la fusión de la televisión e Internet, derivándose de tan extraordinaria hibridación el advenimiento de un nuevo y complejo medio de comunicación, el cual definitivamente podría contribuir a extender el Comercio Electrónico en el mundo de forma todavía más acelerada. El tipo de prácticas informativas y comerciales que podrían realizarse a través de ese nuevo medio de comunicación definitivamente reta la imaginación de cualquiera. Ese año las ventas que se realizarán a través de Internet alcanzarán cifras de trillones de dólares. ActivMedia, por ejemplo, estima que en el año 2003 se realizarán ventas en línea por 1.3 trillones de dólares. ${ }^{21}$ En cambio la firma Forester Research calcula que en ese mismo año las ventas por Internet ascenderán a 3.2 trillones de dólares. ${ }^{22}$

18. Véase Moira Jacobs: "International E-Business Oportunities and Challenges: A Small Business Perspective". Ponencia presentada en The 1999 e-Business Opportunities, Challenges, and Winning Strategies. Universidad de Montana en Missoula y The Montana World Trade Center, Estados Unidos, 2-5 de agosto de 1999.

19. Idem.

20. Vadim Levtin: op. cit.

21. Moira Jacobs: op. cit.

22. Vadim Levtin: op. cit. 
De acuerdo con información de NUA Internet Suveys, en septiembre de 1999 la población mundial que ya tenía acceso a Internet ascendía a 201 millones de personas:

TABLA 1

Usuarios de internet, septiembre, 1999

\begin{tabular}{|l|c|}
\hline \multicolumn{1}{|c|}{ Región } & Millones De usuarios \\
\hline África & 1.72 \\
\hline Asia / Pacífico & 33.61 \\
\hline Europa & 47.15 \\
\hline Medio Oriente & 0.88 \\
\hline Canadá y Estados Unidos & 112.4 \\
\hline América Latina & 5.29 \\
\hline
\end{tabular}

Fuente: NUA Internet Surveys ${ }^{23}$

Sin embargo, en tal solo diez meses; es decir, de septiembre de 1999 a junio del 2000, 121.73 millones de personas se convirtieron en nuevos usuarios de la red. Así en junio del 2000 el total de usuarios de Internet se estimaba ya en 332.73 millones.

TABLA 2

Usuarios de internet, Junio, 2000

\begin{tabular}{|l|c|}
\hline \multicolumn{1}{|c|}{ Región } & MiLlones de usuarios \\
\hline África & 2.77 \\
\hline Asia / Pacífico & 75.5 \\
\hline Europa & 81.82 \\
\hline Medio Oriente & 1.90 \\
\hline Canadá y Estados Unidos & 147.48 \\
\hline América Latina & 13.19 \\
\hline
\end{tabular}

Fuente: NUA Internet Surveys ${ }^{24}$

23. Véase el sitio www de NUA Internet Surveys, el cual reúne valiosa información demográfica sobre Internet en: http://www.mua.ie/surveys.

24. Idem. 
Es interesante observar que durante el periodo comprendido entre septiembre de 1999 y junio del 2000 , el incremento porcentual más pequeño en el número de usuarios de Internet se observó en la región Canadá y Estados Unidos. En cambio en América Latina se registró el incremento porcentual más significativo en el número de usuarios de Internet: 249.34 por ciento.

Respecto al desarrollo alcanzado por el Comercio Electrónico en América Latina, la firma Boston Consulting Group estimó en 1998 que el 88 por ciento de las ventas en línea que se realizarían en América Latina durante 1999 se efectuaría particularmente en Brasil, pues conforme a las estimaciones de Boston Consulting Group, precisamente en ese país se concentraba el 45 por ciento de los cibernautas de América Latina. ${ }^{25}$

\begin{tabular}{|c|c|c|c|c|c|c|c|c|c|}
\hline Pafs & cotdd & Jul/96 & Ene/97 & $J u l / 97$ & Ine/98 & Jul/98 & Ene/99 & Jul/9s & Ene/00 \\
\hline Japón & jp & 46,427 & 734,406 & 955,688 & $1,168,956$ & $1,352,200$ & $1,687,534$ & 2,072529 & $2,636,541$ \\
\hline Reino Unido & uk & 579,492 & 591,624 & 878,215 & 987,733 & $1,190,663$ & $1,423,804$ & $1,599,497$ & $1,901,812$ \\
\hline $\begin{array}{l}\text { Estados } \\
\text { Unidos }\end{array}$ & us & 432,727 & 587,175 & 825,048 & $1,076,583$ & $1,302,204$ & $1,562,391$ & $1,555,882$ & $1,875,663$ \\
\hline Dinamarca & de & 548,168 & 721847 & 875,631 & 994,926 & $1,154,340$ & $1,316,893$ & $1,426,928$ & $1,702,486$ \\
\hline Canade & ca & 424,356 & 603,325 & 690,316 & 839,141 & $1,027,571$ & $1,119,172$ & $1,294,447$ & $1,669,664$ \\
\hline Australia & $\omega$ & 397460 & 514,760 & 707,611 & 665,403 & 750,327 & 792,351 & 907.637 & $1,090,468$ \\
\hline Holunda & nl & 214,704 & 270,521 & 341,560 & 381,172 & 514,660 & 364,129 & 637,591 & 820994 \\
\hline Francin & fr & 189,786 & 245,501 & 292,096 & 333,306 & 431,045 & 488,043 & 653,686 & 779,879 \\
\hline Italia & It & 113,776 & 149,595 & 211,966 & 243,250 & 320,725 & 338,822 & 393,627 & 658,307 \\
\hline Finlandis & 6 & 27207 & 283,526 & 335,956 & 450,044 & 513,527 & 546,244 & 577,029 & 631248 \\
\hline Taiwin & $T w$ & 30,645 & 34,650 & 40,706 & 176,836 & 103,661 & 308,676 & 424,209 & 597,036 \\
\hline Suecia & se & 186,312 & 232,955 & 284,478 & 319,065 & 380,634 & 431,809 & 515,031 & 594,627 \\
\hline Brasil & $\mathrm{Br}$ & 46,854 & 77,148 & 68,685 & 117,200 & 163,890 & 215,086 & 310,138 & 46,444 \\
\hline Espafte & Ea & 62,447 & 110,041 & 121,823 & 168,913 & 243,436 & 264,245 & 302,457 & 415,641 \\
\hline Mexico & Mx & 20,253 & 29,840 & 35,238 & 41,659 & 83,949 & 112,620 & 224,239 & 404,873 \\
\hline
\end{tabular}

Fuente: Internet Software Consortium ${ }^{26}$

25. Moira Jacobs: op. cit.

26. http://www.isc.org 
¿Qué retos impone la economía digital a la próxima administración?

Conforme a la información disponible sobre el número de hosts bajo cada TLD (genéricos como el .com, net y .org y nacionales como .mx, .fr, .ar los cuales se conocen como ccTLDs), en julio de 1999 México ocupaba el vigésimo lugar mundial, con un total de 224,239 hosts. A Brasil le correspondía la décimo cuarta posición, con un total de 310,138 hosts. El primer lugar de los países hispanoparlantes pertenecía a España, país que reportaba un total de 302,457 hosts. España entonces ocupaba la décimo quinta posición mundial. ${ }^{27}$

En enero del 2000 Brasil transitó a la décima tercera posición con un total de 446,444 hosts. España conservó el primer lugar entre los países hispanoparlantes con 415,641 hosts, pasando de la décimo quinta a la décimo cuarta posición. Durante ese mismo periodo México ascendió a la décima quinta posición, con un total de 404,873 hosts, registrando un incremento porcentual mayor al observado por España y Brasil. Ese dato admite interpretarse como un importante indicador del desarrollo que ha alcanzado la industria de Internet en México.

En México, como en la mayor parte de América Latina, a partir de 1999 han proliferado los "portales" orientados al desarrollo de operaciones de Comercio Electrónico, los cuales hoy pueden contarse hasta por decenas. La mayor parte de los portales enfocados al atractivo mercado latinoamericano hasta el día de hoy han enfrentado considerables pérdidas. Tal es el caso de Starmedia, por ejemplo, pues a pesar de que Wall Street aceptó invertir en el financiamiento del portal del uruguayo Fernando Espuelas, éste ha resentido cuantiosas pérdidas. Simplemente cabe señalar que, de acuerdo con información publicada por El Financiero, en el segundo trimestre del año en curso Starmedia reportó pérdidas por 44 millones de dólares. ${ }^{28}$

Es posible aseverar que, entre otros factores, tal situación responde al hecho de que en sus estrategias de mercadotecnia, los propietarios de los grandes portales de la región han resultado incapaces de poder reconocer y distinguir las profundas diferencias culturales que observan los latinoamericanos en sus hábitos de compra, respecto del proceder de los consumidores canadienses o estadounidenses. Las estrategias de mercadotecnia que han ensayado los propietarios de los grandes portales de América Latina en realidad parece que originalmente fueron concebidas para atraer al activo mercado estadounidense.

Además se ha subestimado la necesidad de informar al público consumidor sobre las ventajas que les pudiese reportarle el realizar operaciones de Comercio Electrónico en línea. Los principales esfuerzos comunicativos que hasta ahora se realizan a través de los principales medios masivos para promover las actividades

27. Véase: http://www.nic.nix/esta/survey. html

28. Véase la notas "Buscan socio en EsMas" en El Financiero. "La ruta del dinero", lunes 21 de agosto de 2000. 
comerciales de los portales son de carácter promocional, no informativo. Así, pese a la considerable inversión que en materia de publicidad han realizado los grandes portales de la región, éstos no consiguen incrementar significativamente la masa crítica de consumidores reales, pues hasta ahora no han logrado ganarse su confianza.

Para colmo, a lo largo de la administración del Presidente Ernesto Zedillo, el gobierno de la República ha resultado incapaz de poder articular una estrategia definida que efectivamente contribuya a promover el adecuado desarrollo del Comercio Electrónico en México.

Si bien Bancomext y SECOFI concibieron un proyecto en materia de Comercio Electrónico: “b@ancomext.com Comercio Electrónico Sin Fronteras”, pretendiendo convertirse en el principal punto de referencia en el ciberespacio para las empresas extranjeras que se mostrasen interesadas en efectuar negocios en línea con empresas mexicanas, tal proyecto sólo consideraba a empresas que ya disponían de una "demostrable capacidad exportadora", quedando totalmente al margen de la propuesta las miles de micro, pequeñas y medianas empresas que, pese a generar la mayor parte de los empleos en el país, no disponían de la "demostrable capacidad exportadora" que les exigía Bancomext. ${ }^{29}$

También podría cuestionarse si las empresas que efectivamente disponían de la "demostrable capacidad exportadora" que les exigía Bancomext, efectivamente considerarían el portal de Bancomext y SECOFI como la mejor opción para promover sus actividades comerciales en el ciberespacio. Suponemos que ese limitado grupo de empresas perfectamente podría emprender tal iniciativa por cuenta propia, como efectivamente ha ocurrido.

El adecuado desarrollo del Comercio Electrónico en México demanda profundas transformaciones jurídicas, mismas que resulta indispensable extender mucho más allá de las posibles reformas que admitan los códigos penal, civil y mercantil.

De ninguna manera basta con haber concretado ya algunas reformas a determinados artículos del Código Penal para el Distrito Federal en Materia del Fuero Común y para toda la República en materia de fuero federal. En realidad será indispensable legislar específicamente en materia de Internet y Comercio Electrónico.

Antes de abordar tal tema, procederemos a identificar algunas de las notables contribuciones jurídicas que han realizado, en materia de Comercio Electrónico, algunas de las instituciones internacionales que de alguna manera inciden en la regulación de Internet.

29. Presentación en Power Point en el "Séptimo Congreso del Comercio Exterior Mexicano, evento que se realizó el 12 de noviembre de 1999 en Mérida, Yucatán. En ese acto Enrique Vilatela Riva presentó el proyecto "b@ncomext.com. Comercio Electrónico Sin Fronteras". 


\section{¿Cuáles son los organismos internacionales que intervienen en el desarrollo del Comercio Electrónico?}

Entre los principales organismos internacionales que ejercen algunas regulaciones sobre el desarrollo de Internet y del Comercio Electrónico se encuentran: la Organización Mundial de la Propiedad Intelectual (OMPI-wIPo); la Comisión de las Naciones Unidas para el Derecho Mercantil Internacional (CNUDMI-UNCITRAL); la Organización para la Cooperación y el Desarrollo Económico (OCDE-OECD); el Área de Libre Comercio de las Américas (ALCA); y la Cámara de Comercio Internacional (CCI-ICC). A continuación describiremos cuáles son las funciones que desempeñan esos organismo en materia de Internet y Comercio Electrónico.

\section{Organización Mundial de la Propiedad Intelectual (OMPI-WIPO)}

La Organización Mundial de la Propiedad Intelectual (OMPI) es una institución intergubernamental cuya sede se encuentra en la ciudad de Ginebra, Suiza. La OMPI es uno de los 16 organismos especializados del sistema de las Naciones Unidas. Su función principal es promover la protección de la propiedad intelectual en el mundo mediante la cooperación de los Estados, y administrar varios tratados multilaterales que aseguren los aspectos jurídicos y administrativos que supone el respeto a la propiedad intelectual.

De acuerdo con la OMPI, la propiedad intelectual comprende dos ramas principales:

- La propiedad industrial (especialmente las invenciones, marcas de fábrica y de comercio, dibujos y modelos industriales y denominaciones de origen).

- El derecho de autor (especialmente las obras literarias, musicales, artísticas, fotográficas y audiovisuales).

Una considerable cantidad de los recursos de que dispone la OMPI están destinados a fomentar la cooperación con los países en desărollo. ${ }^{30}$

En materia de Internet la OMPI ha realizado recomendaciones relativas a cuestiones de propiedad intelectual relacionadas con los nombres de dominio de Internet, incluyendo la propuestas de solución a posibles controversias. ${ }^{31}$

30. Véase: http://www.wipo.org/eng/ratific/c-wipo.htm.

31. Las recomendaciones de la OMPI relativas a la situación de los nombres de los dominios en Internet estarán disponibles para la Internet Corporation for Assigned Names and Numbers (ICANN), la nueva organización que se encargará de administrar el Sistema de Nombres de Dominio en Internet. 
A través de su sitio Web (http://www,wipo.org), la OMPI recaba información respecto al programa de trabajo y las actividades relativas a la propiedad intelectual y al Comercio Electrónico. Ese sitio publica su información en inglés, francés y español además de ofrecer enlaces al Programa Digital de la OMPI —disponible en inglés, árabe, chino, francés, español y ruso-, el cual establece una serie de pautas y objetivos para la OMPI en la búsqueda de soluciones prácticas a los desafios planteados por la repercusión del Comercio Electrónico sobre los derechos de propiedad intelectual.

El sitio WWW además concentra valiosa información respecto al delicado tema jurídico de los Nombres de los Dominio en Internet. Además en la sección "Otras actividades de programa" ofrecen información sobre las actividades que realiza la OMPI en materia de Comercio Electrónico. Además de publicar este tipo de información en su sitio web, la OMPI distribuye documentos en materia de Comercio Electrónico e Internet por correo vía del electrónico y también en papel. Para la distribución por correo electrónico se debe tramitar la petición correspondiente en la siguiente dirección: process@wipo2.wipo.int

También en ese sitio wwW es posible consultar el Manual sobre Comercio Electrónico y Propiedad Intelectual que la OMPI publicó en mayo de 2000, en el cual se abordan las principales cuestiones relacionadas con el Comercio Electrónico y sus repercusiones en materia de propiedad intelectual. También es posible consultar en línea la carpeta de información de la OMPI sobre Comercio Electrónico, la cual contiene todos los documentos de la OMPI relacionados con el tema. ${ }^{32}$

Comisión de las Naciones Unidas para el Derecho Mercantil Internacional (CNUDMI-UNCITRAL)

La Comisión de las Naciones Unidas para el Derecho Mercantil Internacional (UNCITRAL, por sus siglas en inglés), fue establecida por la Asamblea General en 1966 (resolución 2205 XXI, del 17 diciembre de 1966). Según la propia UNCITRAL, la Asamblea General, al establecer la Comisión, reconoció que las disparidades entre las legislaciones nacionales que regulan el comercio internacional crean algunos obstáculos al desarrollo del comercio, y consideró que la Comisión representaría la instancia idónea para que las Naciones Unidas pudiesen desempeñar un papel más activo en la reducción o en la eliminación de esos obstáculos.

32. Para mayor información puede consultarse la siguiente dirección: http://ecommerce.wipo.int/index-es.html. 
La Asamblea General de Naciones Unidas encomendó a la Comisión (UNCITRAL) la tarea de impulsar la progresiva unificación del derecho mercantil internacional. A partir de ello la UNCITRAL se ha convertido en el principal órgano jurídico del sistema de las Naciones Unidas en la esfera del derecho mercantil internacional.

La Comisión se encuentra integrada por 36 Estados miembros, los cuales fueron elegidos a través la Asamblea General. Su composición afirman que es representativa de las diversas regiones geográficas y de los principales sistemas jurídicos y económicos del mundo. Los miembros de la Comisión se eligen por periodos de seis años y cada tres años expira el mandato de la mitad de ellos.

Las principales áreas de trabajo de la UNCITRAL son:

- Operaciones de compraventa internacional y operaciones conexas.

- Transporte internacional de las mercancías.

- Arbitraje y conciliación comercial internacional.

- Contratación pública.

- Contratos de construcción.

- Pagos internacionales.

- Insolvencia transfronteriza.

- Comercio Electrónico.

En el área relativa al Comercio Electrónico precisamente destaca la Ley Modelo de la CNUDMI sobre Comercio Electrónico. Esa ley, elaborada en el año de 1996, tiene por objeto facilitar el uso de avanzadas tecnologías de comunicación y de almacenamiento de información en la práctica de las operaciones comerciales en línea, considerando, por ejemplo, disposiciones en materia de intercambio electrónico de datos (EDI), correo electrónico y la telecopia con o sin el soporte de Internet.

La Ley Modelo además incorpora algunos criterios para apreciar el valor jurídico de los mensajes electrónicos, y por tal motivo representa una importante contribución al desarrollo de las comunicaciones en línea que se realizan sin el uso del papel.

Como complemento de las normas generales, la Ley contiene también una serie de lineamientos para el desarrollo del Comercio Electrónico en áreas específicas, como sería precisamente el transporte de las mercancías. Con el propósito de poder asistir a los legisladores, la Comisión además ha elaborado una Guía para la Incorporación de la Ley Modelo de la CNUDMI sobre Comercio Electrónico al derecho interno. ${ }^{33}$

33. Véase http://www.uncitral.org. 
Organización para la Cooperación y el Desarrollo Económico (OECD)

La Organización para la Cooperación y el Desarrollo Económico (OECD) se encuentra integrada por 29 países, entre los que cabe mencionar a Japón, Australia, Nueva Zelanda, Finlandia, México, República Checa, Hungría, Polonia y Corea. Ese organismo proporciona información especializada a los gobiernos sobre temas de desarrollo, economía y política social. La OECD compara experiencias, busca soluciones a problemas comunes y trabaja conjuntamente en la coordinación de políticas locales e internacionales. Los países afiliados sólo deben cumplir con dos requisitos: contar con una economía de mercados abiertos e instituciones democráticas.

La OECD recolecta datos, analiza escenarios, estudia tendencias e investiga el desarrollo de la economía mundial para posteriormente publicar sus reportes y remitirlos a los países afiliados.

La OECD afirma que los gobiernos necesitan adaptar sus marcos jurídicos para estimular el adecuado desarrollo de nuevos canales para el comercio mundial. La OECD se encuentra examinado las implicaciones del Comercio Electrónico en áreas críticas de la microeconomía, como son la aplicación de impuestos, el desarrollo de prácticas fraudulentas en línea, la necesidad de garantizar la protección del consumidor, el respeto a la privacidad y las condiciones de seguridad que requieren las operaciones comerciales en línea. Además la OECD investiga las consecuencias económicas y sociales que se desprenden del desarrollo del Comercio Electrónico sobre esferas de interés público internacional como son el trabajo, la educación y la salud.

La OECD ha promovido entre los representantes de gobiernos, empresas, sindicatos y otras organizaciones internacionales, el desarrollo conjunto de una serie de estrategias que contribuyan al mejor desarrollo del Comercio Electrónico.

\section{Área de Libre Comercio de las Américas (ALCA)}

El esfuerzo por unificar las economías del Hemisferio Occidental en un solo acuerdo de libre comercio fue iniciado en la Cumbre de las Américas, llevada a cabo en diciembre de 1994 en la ciudad de Miami. Los mandatarios de las 34 democracias en la región acordaron establecer un "área de libre comercio de las Américas" (ALCA) y concluir las negociaciones para el acuerdo antes del año 2005. Asimismo, los líderes se comprometieron a lograr un avance sustancial hacia el ALCA para el año 2000. Sus decisiones se encuentran en la Declaración de Principios y el Plan de Acción de la Cumbre. ${ }^{34}$

34. Véase: http://www.alca-ftaa.org/alca_s.asp 
¿Qué retos impone la economía digital a la próxima administración?

De acuerdo con la información que publican en su sitio Web, El esfuerzo por establecer el Área de Libre Comercio de las Américas (ALCA), es un proceso dinámico que involucra la participación de tres actores básicos:

- Los Ministros de Comercio del Hemisferio Occidental que han desarrollado el plan de trabajo completo para el ALCA.

- Los 12 grupos de trabajo del ALCA establecidos por los Ministros de Comercio, que recolectan y recopilan información del estado actual de las relaciones comerciales en el Hemisferio.

- Los Viceministros de Comercio del Hemisferio Occidental, quienes coordinan los esfuerzos de los grupos de trabajo y asesoran a los Ministros de Comercio.

Los 12 Grupos de Trabajo se han reunido en diferentes países de América. Cada uno de los grupos, bajo la dirección de los Ministros de Comercio, se ha dedicado a recabar información sobre la situación que presenta el desarrollo de las relaciones comerciales en sus respectivas áreas, con el propósito de determinar posibles vías de negociación.

Según la ALCA, los Ministros instruyeron a sus Viceministros a recibir las recomendaciones de los Grupos de Trabajo, para poder así dirigir, evaluar y coordinar el trabajo de los grupos. Así la ambiciosa meta de crear un área de libre comercio de las Américas recibió un importante impulso con la Declaración Ministerial de San José, el 19 de marzo de 1998. Ese documento, acordado por los Ministros de Comercio de las 34 democracias participantes en el proceso del ALCA, ha servido de base para la puesta en marcha formal de las negociaciones hemisféricas llevadas a cabo por los Jefes de Estado que se reunieron en Santiago de Chile los días 18 y 19 de abril de 1998. La Declaración de San José representa el compromiso de 34 países para la realización del esquema más ambicioso de liberalización comercial desde la época de la Ronda Uruguay. Implica, asimismo, el esfuerzo más importante de integración regional llevado a cabo entre países desarrollados y países en vías de desarrollo, con el objetivo común de lograr el libre comercio y la inversión en bienes y servicios.

Las negociaciones del ALCA incluyen temas como el establecimiento de un régimen común de inversiones, compras al sector público y políticas de competencia que no se encuentran actualmente consideradas bajo el marco de la $\mathrm{OMC}$, y que aún no forman parte de los acuerdos existentes entre un gran número de países. Además se examinan temas de interés general para las naciones del hemisferio, como la agricultura y el acceso a los mercados, los servicios y sistemas de inversión, las 
políticas de competencia y los subsidios, medidas antidumping y derechos compensatorios.

De acuerdo con los representantes del ALCA, las diferencias en los niveles de desarrollo y el tamaño de las economías participantes serán tomados en consideración durante las negociaciones para asegurar que los países más pequeños del hemisferio igualmente se beneficien de la gran apertura comercial. Mientras se efectúen las negociaciones, los representantes de la sociedad civil tendrán la posibilidad de presentar sus opiniones sobre los temas a tratarse. Un comité de representantes gubernamentales servirá de enlace para el intercambio de información entre aquellos sectores de la sociedad interesados en participar y los negociadores de los diversos temas.

En materia de Internet se ha designado una comisión especial, denominada “ $\mathrm{Co}$ mité Conjunto de Expertos del Sector Público y Privado en Comercio Electrónico", la cual se encargará de elaborar recomendaciones a los Ministros de Comercio sobre cómo deben aumentarse y ampliarse los beneficios del Comercio Electrónico y, en particular, sobre cómo debería tratarse el Comercio Electrónico en el contexto de las negociaciones del ALCA.

Cámara de Comercio Internacional (ICC)

La Cámara de Comercio Internacional (ICC) es un organismo empresarial internacional que promueve el desarrollo de un sistema abierto de inversiones y de comercio internacional, impulsando la apertura de las economías de mercado.

La ICC sostiene la tesis de que el comercio es una fuerza poderosa para la paz y la prosperidad. Los origenes de esta organización pueden establecerse a principios de siglo. El pequeño grupo de líderes empresariales que fundaron la ICc adoptó el nombre de "los mercaderes de la paz". Puesto que las empresas y asociaciones afiliadas practican el comercio internacional, la ICC tiene una autoridad indiscutible a la hora de establecer normas que regulen los intercambios fronterizos. Tales normas, aunque voluntarias, son observadas diariamente en un amplio número de operaciones comerciales en todo el mundo.

$\mathrm{Al}$ año de haberse consumado la fundación de las Organización Naciones Unidas, a la ICC se le otorgó el rango de organismo consultivo del más alto nivel ante la ONU y sus organismos especializadas. Los expertos de la ICC establecen sus posturas sobre temas de política internacional, comercio e inversiones, servicios financieros, el desarrollo y las aplicaciones de las tecnologías de la información, las telecomunicaciones, ética del marketing, medio ambiente, transporte, leyes de la compe- 
tencia y propiedad intelectual, entre otros. Actualmente la ICC agrupa a miles de miembros en más de 130 países.

La ICC promueve e informa a sus asociados sobre diferentes aspectos tecnológicos y de Comercio Electrónico como los siguientes:

- Telecomunicaciones y Tecnologías de Información.

- Proyecto Comercio Electrónico. Desarrollado para crear confianza a nivel global en lo que se refiere a transacciones electrónicas, definiendo lo mejor posible las prácticas de negocio en la era digital

- Guías, códigos y reglamentos.

- Cláusulas modelo para el uso en contratos comerciales internacionales.

- GUIDEC. Guía General para el uso internacional del Comercio Electrónico.

- Políticas y reportes generales.

- Plan Global de acción para el Comercio Electrónico.

- Sobre la administración de nombres de dominio y direcciones electrónicas.

- Confidencialidad, Privacidad, Protección de datos en negocios electrónicos.

- Autorregulación, o regulación y cooperación para la era digital.

- Protección de marcas en el Comercio Electrónico

Todos los organismos descritos anteriormente han demostrado tener cierta influencia en el desarrollo de Internet y el Comercio Electrónico. Cada uno actúa de acuerdo a su área de competencia con un propósito firme y común: no obstaculizar el desarrollo de Internet en una escala global y obtener los mayores beneficios del adecuado aprovechamiento de las tecnologías de información para elevar la calidad del nivel de vida en el mundo.

\section{¿Cuál es el alcance de las reformas dispuestas} en materia de Comercio Electrónico en México?

El 29 de mayo del 2000, el Presidente de los Estados Unidos Mexicanos, doctor Ernesto Zedillo Ponce de León dio a conocer, a través del Diario Oficial de la Federación, diversas reformas y adiciones a la legislación federal en materia de Comercio Electrónico. Los cambios y anexos modificaron algunas disposiciones contenidas en el Código Civil para el Distrito Federal en Materia Común y para toda la República en Materia Federal, así como en el Código Federal de Procedimientos 
Civiles, el Código de Comercio, y la Ley Federal de Protección al Consumidor. Nueve días después entró en vigor el decreto que representa el primer marco legal en México para el desarrollo del Comercio Electrónico.

Los razonamientos que impulsaron el desarrollo de las nuevas reformas y adiciones en materia de Comercio Electrónico obedecieron a la delicada ausencia de un ordenamiento jurídico que expresamente reconociese en México la validez de las transacciones que se efectúan en el ciberespacio.

De acuerdo con el dictamen de las comisiones legislativas que en la Cámara de Diputados estuvieron involucradas en el desarrollo de las reformas y adiciones a la legislación federal, entre las consideraciones más importantes para la creación del marco jurídico sobre el Comercio Electrónico destacaron las siguientes:

- Los distintos avances en la electrónica y su consecuente transformación en las formas de operación de las sociedades: trabajo, aprendizaje y comunicación.

- Las redes de información y la transformación de los hábitos de las sociedades, destacando principalmente las nuevas formas de operación de las empresas: las organizaciones integradas.

- La evidencia internacional de cómo las tecnologías de la información contribuyen a desarrollo de la productividad de las empresas.

- La comprensión del Comercio Electrónico como un elemento que facilitará, al sector productivo de nuestro país, el aprovechamiento de la revolución informática actual que representa una poderosa estrategia para el impulso de la competitividad y eficiencia de las empresas mexicanas de cualquier dimensión.

- El crecimiento del la infraestructura tecnológica nacional: En 1997 había en nuestro país cerca de 3.5 millones de computadoras, para finales de 1999 la cifra superó los 4.2 millones.

- El crecimiento relativo al número de usuarios de Internet: Actualmente se estima que el mercado de Internet, en nuestro país, supera a los 3 millones de usuarios con un crecimiento de 65 por ciento, una de las tasas de crecimiento más alto a nivel internacional.

- La modernización de las empresas mexicanas: El 70 por ciento de las operaciones de Comercio Electrónico se realizan bajo el modelo de negocios B2B.

El número de empresas en México que ya realizan transacciones a través de medios electrónicos asciende a más de 4,000. La mayoría todavía utiliza el intercambio electrónico de datos (EDI), pero en un futuro no muy distante es seguro que estarán realizando transacciones a través de Internet. 
¿Qué retos impone la economía digital a la próxima administración?

A través de las avanzadas tecnologías de comunicaciones asociadas a Internet, un corporativo que realiza operaciones en distintas naciones fácilmente puede establecer un extenso y eficiente sistema de comunicaciones entre cada una de sus sucursales, e incluso puede llegar a extenderlo a sus proveedores y otros participantes de la cadena productiva. A esa extensión de la red organizacional se le conoce como Extranet. Con base en estas redes interorganizacionales se crean nuevas formas de operación y desarrollo del trabajo en las empresas, pero sin la presencia de un marco jurídico idóneo, las posibilidades de los sistemas extendidos de información adquieren el rango de predicamentos para la empresa.

De acuerdo con la Exposición de Motivos de la iniciativa de reformas y adiciones a la legislación federal (http://gaceta.cddhcu.gob.mx/Gaceta/2000/abr/ 20000426.html), en materia de Comercio Electrónico, el gobierno asume un rol muy importante en la promoción y desarrollo de las tecnologías de información para el mejoramiento del servicio a los usuarios. El empleo de avanzados sistemas de información no sólo hace más eficientes las relaciones entre gobierno, empresas y ciudadanía en general; también admite un impacto positivo en la economía del país.

Antes de que entrase en vigor el nuevo marco jurídico en materia de Comercio Electrónico, la legislación mexicana se limitaba a prever como únicos medios de comunicación para la contratación entre no presentes al correo y al telégrafo. Como consecuencia de tal disposición, las partes de un contrato tenían la opción de sujetarse a un mecanismo que reconociera el uso de medios electrónicos para dar el consentimiento, siempre y cuando existiera la previa celebración de un contrato marco por escrito. Mediante este recurso se podía evitar la repudiación o violación de las obligaciones contraídas por las partes; sin embargo, el uso de los medios electrónicos estaba limitado a lo exclusivamente previsto en el contrato marco, y podía surgir la necesidad de adicionarlo o celebrar uno nuevo para cualquier modalidad de las obligaciones originalmente contraídas.

En términos generales ninguna ley reconocía el uso de los medios electrónicos de manera universal, antes de las reformas difundidas el 29 de mayo. En caso de un litigio, el juez o tribunal tenía que allegarse de medios de prueba indirectos para determinar que una operación realizada por medios electrónicos era o no válida. De acuerdo a la Exposición de Motivos para el desarrollo de estas nuevas reformas y adiciones a la legislación federal, la situación había originado que diversas empresas frenaran sus inversiones orientadas a realizar transacciones por medios electrónicos como consecuencia de la incertidumbre legal en caso de controversias.

Como señalamos ya con anterioridad, en el ámbito internacional, la Comisión de las Naciones Unidas para el Derecho Mercantil Internacional (UNCITRAL) ha he- 
cho importantes esfuerzos jurídicos para regular el Comercio Electrónico. Del trabajo de esta comisión internacional derivó la "Ley Modelo sobre Comercio Electrónico", propuesta a todos los Estados como guía para establecer o fortalecer la legislación que normaría el uso de métodos de comunicación y almacenamiento de información sustitutivos del papel, y con ello daría valor jurídico a la utilización de los nuevos medios digitales.

Por lo anterior, las comisiones involucradas en el desarrollo de la iniciativa de reformas y adiciones a la legislación federal en materia de Comercio Electrónico consideraron que el sistema jurídico mexicano debería incluir las menciones necesarias para aprovechar los avances logrados no sólo en el ámbito comercial, sino también en otros sectores que podían ser afectados por este avance tecnológico, de tal manera que así pudiera lograrse una interacción entre todos los campos, considerando el impacto en su conjunto y no de manera aislada. El resultado concreto fueron las reformas y adiciones a diversas disposiciones de la legislación federal en materia de Comercio Electrónico.

La información destacada de las reformas realizadas a diversas leyes de la República Mexicana en materia de Comercio Electrónico, se puede resumir de la siguiente forma: en lo que respecta a diversas reformas y adiciones al Código Civil para el Distrito Federal en materia común y para toda la República en materia Federal, sobresalen las siguientes disposiciones:

1. Modificación de la denominación del Código Civil para el Distrito Federal en Materia Común y para toda la República en Materia Federal, afectando directamente a su artículo primero, el cual queda de la siguiente manera: "Las disposiciones de este Código regirán en toda la República en asuntos del orden federal" (Código Civil Federal).

2. Sobre la anexión al artículo 1803 , referente al consentimiento en los contratos, el cual ahora se considera expreso cuando la voluntad se manifiesta verbalmente, por escrito, por medios electrónicos, ópticos o por cualquier otra tecnología, o por signos inequivocos. ${ }^{35}$

3. Sobre la modificación del artículo 1805 , referente al perfeccionamiento del contrato, el cual queda de la siguiente manera: "Cuando la oferta se haga a una persona presente, sin fijación de plazo para aceptarla, el autor de la oferta queda desligado si la aceptación no se hace inmediatamente. La misma regla se aplicará a la oferta hecha por teléfono o a través de cualquier otro medio electrónico,

35. El subrayado corresponde a las adiciones o modificaciones introducidas. 
¿Qué retos impone la economía digital a la próxima administración?

óptico o de cualquier otra tecnología que permita la expresión de la oferta y la aceptación de ésta en forma inmediata".

4. Del artículo 1811 sobresale el aspecto del no requerimiento de estipulación previa entre los contratantes para que produzca efectos una propuesta y aceptación hechas a través de cualquier medio electrónico u otra tecnología.

5. Además del Artículo 1834 bis se anexa que los supuestos previstos por el artículo anterior se tendrán por cumplidos mediante la utilización de medios electrónicos, ópticos o de cualquier otra tecnología.

Sobre el Código Federal de Procedimientos Civiles destacan los siguientes cambios:

1. En el artículo segundo se adiciona el artículo 210-A para quedar de la siguiente manera: "Se reconoce como prueba la información generada o comunicada que conste en medios electrónicos, ópticos o en cualquier otra tecnología (210-A)". Por lo tanto se aplica a tecnologías como el correo electrónico, grupos de discusión, chats y páginas Web.

2. En el artículo tercero aparecen las siguientes reformas al 18, 20, 21 párrafo primero, 22, 23, 24, 25, 26, 27, 30, 31, 32, 49, 80 y 1205, y se adicionan los artículos 20 bis, 21 bis, 21 bis 1,30 bis, 30 bis 1 y 32 bis $1298-A$; el Título II que se denomina "Del Comercio Electrónico", y comprende del artículo 89 al 94 . Además se modifica la denominación del Libro Segundo del Código de Comercio.

Principalmente del título II denominado del Comercio Electrónico sobresalen las siguientes modificaciones:

1. Artículo 89. Textualmente dice: "En los actos de comercio podrán emplearse los medios electrónicos, ópticos o cualquier otra tecnología. Para efecto del presente Código, a la información generada, enviada, recibida, archivada o comunicada a través de dichos medios se le denominará mensaje de datos." A partir de esta disposición se describen los aspectos referentes a: la procedencia del mensaje de datos (artículo 90), la recepción de la información (artículo 91), el acuse de recibo (artículo 92), la exigencia de la forma escrita para los contratos (artículo 93), el lugar de expedición del mensaje de datos (artículo 94), pruebas admisibles (artículo 1205), los mensajes de datos como prueba (artículo 1298-A).

Con base en esta serie de artículos se reconoce y legaliza distintos aspectos importantes del proceso de Comercio Electrónico que actualmente rige en nuestro país. 
En el caso de la Ley Federal de Protección al Consumidor destacan los siguientes puntos:

1. Fracción VIII del artículo primero, pretende la efectiva protección al consumidor en las transacciones efectuadas a través del uso de medios electrónicos, ópticos o de cualquier otra tecnología y la adecuada utilización de los datos aportados.

2. Del artículo 24, fracción IX bis referente a la promoción, difusión y uso de códigos de ética, por parte de proveedores, que incorporen los principios previstos por la Ley Federal de Protección al Consumidor con respecto a las transacciones que celebren con consumidores a través del uso de medios electrónicos, ópticos o de cualquier otra tecnología;

3. Del Capítulo VIII Bis referente a los derechos de los consumidores en las transacciones efectuadas a través del uso de medios electrónicos, ópticos o de cualquier otra tecnología sobresalen los siguientes aspectos: la aplicación de las disposiciones del capítulo a las relaciones entre proveedores y consumidores en las transacciones efectuadas a través del uso de medios electrónicos, ópticos o de cualquier otra tecnología (artículo 76 bis).

Hasta aquí es importante señalar que, aunque es notable el alcance de las nuevas reformas y adiciones a diversas normas que regulan la actividad comercial para los participantes involucrados en el proceso transaccional, quedan todavía algunos aspectos relevantes por definir con mayor claridad. Uno de ellos, el asunto de la privacidad $v s$ la libertad de expresión, el cual queda fuera de los cambios presentados - en ninguna de las modificaciones se observa el tratamiento de tan importante tema. Por otra parte se encuentra el asunto de los crímenes electrónicos, que de alguna manera se encuentra ya legislado en el Código Penal, pero con un tratamiento superficial; y lo referente al derecho a la información, entre otros temas más. Una de las cuestiones más importantes sin resolver aún en estas iniciativas es definir el órgano que se encargará de la vigilancia de las nuevas disposiciones en materia de Comercio Electrónico, pues los órganos actuales no tienen la capacidad para cumplir con esta labor.

Ted Gaebler - autor del texto Reinventing the Government- afirma: "La tecnología está estimulando la necesidad de reinvención de los gobiernos". Específicamente las tecnologías de Internet apoyan los procesos de redefinición del sector público, porque a través de sus diversas herramientas se facilita la creación de nuevas formas de trabajo y organización. Como consecuencia de la 
implementación de nuevas estrategias basadas en el uso de las avanzadas tecnologías de comunicaciones y la operación de un marco jurídico que regule las actividades en el ámbito del Comercio Electrónico, algunos gobiemos han logrado reducir muchos de sus gastos y ofrecer mejores y nuevos servicios a los ciudadanos.

A marchas forzadas la LVII Legislatura finalmente logró introducir las primeras iniciativas orientadas a regular el desarrollo del Comercio Electrónico en México. Tales iniciativas definitivamente nos parecen todavía insuficientes. La próxima administración tendrá que diseñar estrategias definidas en materia de Comercio Electrónico, pues éste podría generar un considerable número de divisas para el país, derivándose la creación de nuevas fuentes de empleo para miles de mexicanos. Por tal motivo resultará indispensable ampliar el marco normativo que habrá de regular el desarrollo del Comercio Electrónico en México.

\section{Bibliografía}

Chomsky Noam (1997), Secretos, mentiras y democracia. Entrevista a Noam Chomsky por David Barsamian, Siglo xxi Editores, México.

Código Civil Para el Distrito Federal en Materia Comun y Para Toda la Republica en Materia Federal (última actualización: 18 de mayo de 2000) http:// www.cddhcu.gob.mx/leyinfo/2/index.htm. Fecha de consulta: 20 de junio de 2000. Código de Comercio (última actualización:18 de mayo de 2000) http:// www.cddhcu.gob.mx/leyinfo/3/index.htm. Fecha de consulta: 20 de junio de 2000. Código Penal Federal (última actualización: 18 de mayo de 2000) http:// www.cddhcu.gob.mx/leyinfo/11/index.htm. Fecha de consulta: 20 de junio de 2000. Davis, Stan y Meyer, Christopher (1999), Blur, the speed of change in the connected economy, Emst \& Young Center for Business Innovation, Editorial Warner Books, Estados Unidos.

De las Comisiones Unidas de Justicia y de Comercio, con Proyecto de Decreto por el que se Dictaminan Diversas Reformas y Adiciones al Código Civil Federal, al Código de Comercio y a la Ley Federal de Proteccion al Consumidor en Materia de Comercio Electrónico http://gaceta.cddhcu.gob.mx/Gaceta/2000/ abr/20000426.html. Fecha de consulta: 20 de junio de 2000.

Dery, Mark (1998), Velocidad de escape. La cibercultura en el final del siglo. Editorial Ciruela, 1998.

Flores Olea, Víctor y Gaspar de Alba, Rosa Elena (1998): Internet y la revolución cibernética, México. 
Foucault, Michell (1985), Vigilar y castigar. Siglo xxi. México.

Gabler, T., (octubre, 1997), "Paradigmas acorralan la reinvención, del Gobierno". Gobierno Digital, Vol. 2, No. 18, p. 9.

Gates, Bill (1999), Business@ @ the speed of thought. Using a digital nervous system, Warner Books, Estados Unidos, 1999.

Holtz, Herman (1999), The consultant's guide to getting business on the Internet. How to network for clients and business opportunities. John Wiley \& Sons, Estados Unidos.

Harmon Roy L (1996), Reinventing the Business, The Free Press, Estados Unidos. Marcuse, Herbert (1986): El hombre unidimensional. Origen, Planeta, Colección Obras Maestras del Pensamiento Contemporáneo. México.

McLuhan Marshall (1985), La comprensión de los medios como extensiones del hombre. Editorial Diana. México.

Negroponte, Nicholas (1996), Ser Digital, Atlántida Océano, México.

Queau, Philipe (1998), Lo virtual. Virtudes y vértigos. Editorial Paidós, España. Secretaria De Comercio y Fomento Industrial. Decreto por el que se reforman y adicionan diversas disposiciones del Código Civil para el Distrito Federal en Materia Común y para toda la República en Materia Federal, del Código Federal de Procedimientos Civiles, del Código de Comercio y de la Ley Federal de Protección al Consumidor. Diario Oficial de la Federación, 29 de mayo de 2000. 